\title{
Introduction to Special Issue on Primate Hybridization and Hybrid Zones
}

\author{
Liliana Cortés-Ortiz ${ }^{1}$ (D) $\cdot{\text { Christian } \text { Roos }^{2} \cdot \text { Dietmar }^{2} \text { inner }}^{3}$
}

Received: 29 November 2018 / Accepted: 30 November 2018 / Published online: 4 February 2019

(C) Springer Science+Business Media, LLC, part of Springer Nature 2019

Hybridization, or interbreeding between individuals of genetically distinct taxa that produces at least some viable offspring, is a natural process that may occur at different stages in the evolution of diverging lineages. The importance of hybridization in the evolutionary history of primates is now well accepted (Arnold and Meyer 2006; Tung and Barreiro 2017; Zinner et al. 2011). Hybridization has been observed or inferred in all major primate lineages, and the outcomes of ancient and current admixture in human and nonhuman primate taxa have had important evolutionary consequences (e.g., Racimo et al. 2015; Tung et al. 2012). However, studies of nonhuman primate hybridization and hybrid zones are still scarce. This Special Issue presents original work on a diverse set of topics related to the study of ancient and current hybridization in primates that originated from a Symposium held during the 26th Congress of the International Primatological Society in Chicago, USA, in August 2016. For this symposium we invited a number of researchers studying hybridization using phylogenetic, behavioral, ecological, or genomic approaches to exchange experiences and approaches in studying primate hybridization, and to highlight ongoing research aimed at understanding the processes and outcomes of hybridization in primates.

Natural hybridization may have various evolutionary outcomes (Fig. 1). If hybrids are sterile or less fit than the parental species, hybridization may have no evolutionary effect (although the fitness of the parent individuals is reduced), or could lead to the emergence of a narrow hybrid zone (tension zone model: Barton and Hewitt 1985; Ridley 2004) with some exchange of alleles between the gene pools of the parental taxa (i.e., introgression). In such zones, interbreeding between hybrid individuals and

$\underline{\text { Handling Editor: Joanna M. Setchell }}$

Liliana Cortés-Ortiz

lcortes@umich.edu

1 Department of Ecology and Evolutionary Biology, University of Michigan, Ann Arbor, MI 48103, USA

2 Gene Bank of Primates and Primate Genetics Laboratory, German Primate Center, Leibniz Institute for Primate Research, 37077 Göttingen, Germany

3 Cognitive Ethology Laboratory, German Primate Center, Leibniz Institute for Primate Research, 37077 Göttingen, Germany 


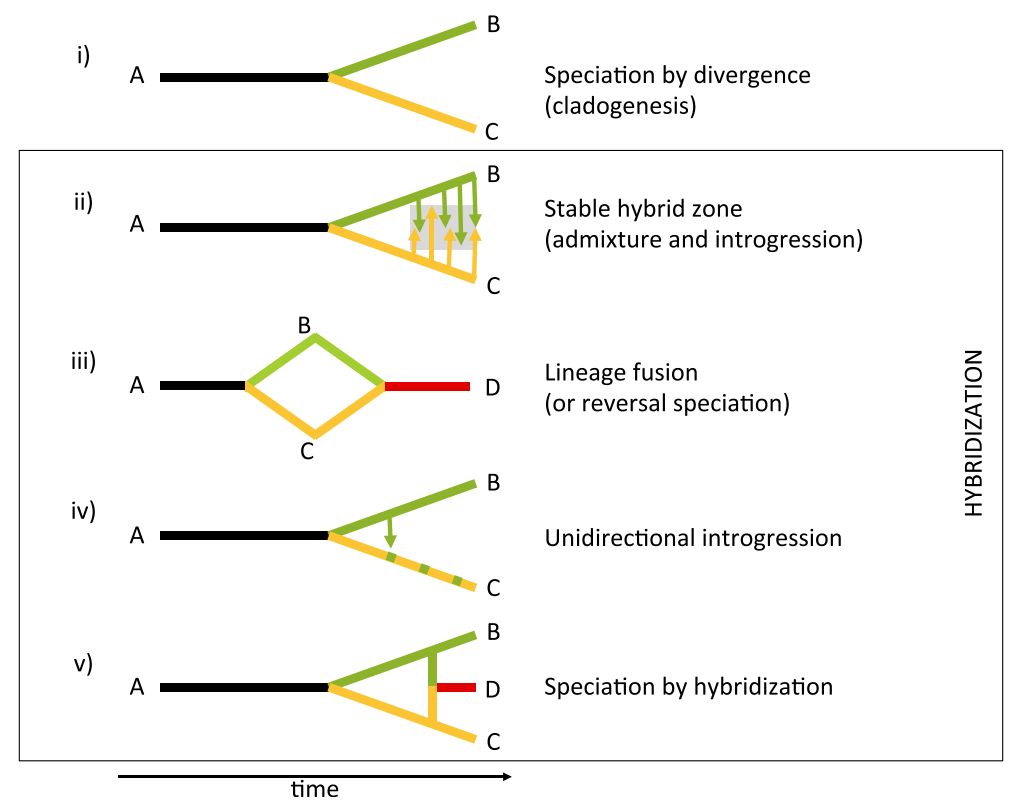

Fig. 1 The formation of a species by the splitting of a lineage into two (i) and possible evolutionary outcomes of hybridization (ii-v). A-D represent different taxa. ii Formation of a hybrid zone with some exchange of alleles (introgression) between parental species. iii Fusion of two divergent lineages into a single lineage or species through hybridization and backcrossing. iv Unidirectional introgression in which only one species receives genetic input from another. $\mathbf{v}$ Formation of a new species by hybridization where hybrid individuals mate assortatively (i.e., do not backcross with individuals of the parental taxa).

backcrossing with parental taxa can lead to the formation of a genetically and phenotypically highly variable population (e.g., the hybrid zone between olive and hamadryas baboons in the Awash Valley, Ethiopia: Bergey 2015; Jolly 1993). Hybridization could also lead to the fusion of the two parental species (termed "speciation reversal": Seehausen 2006) if there is a change in the balance between selection and gene flow; for example, if hybrids have higher fitness than one or both parental species and backcross in both directions, or if species evolved as a byproduct of divergent adaptation and a change in the environment eliminates the ecological restrictions to gene flow between them (Kearns et al. 2018). However, if hybrids do not backcross and show assortative mating among them, a new genetic lineage might evolve leading to a new species (Abbott et al. 2013). Research on hybridization can be broadly divided into studies that investigate ancient events of admixture between diverging taxa that allowed the introgression of alleles into the heterospecific genome and studies that aim to understand the mechanisms that drive hybridization in the present time, and the factors that shape the outcomes of the hybridization process.

Hybridization may also have implications for primate conservation, especially if induced by human activities. In a natural situation, if two formerly allopatric species gain secondary contact and fuse through hybridization, or if one species experiences intense introgression and nuclear swamping, primate diversity will decrease. Anthropogenic activities can also initiate or intensify the hybridization process. For example, the introduction of species in areas outside their natural range may instigate hybridization with an autochthonous species (Callithrix southeastern Brazil; Malukiewicz et al. 
2015). Similarly, a decrease in population densities of closely related sympatric species due to hunting and habitat fragmentation may impel hybridization because of the difficulty of finding conspecific mates (Detwiler et al. 2005). When a hybrid population is established it raises the question of whether or not it should receive the same conservation attentention as other populations (Allendorf et al. 2001; Todesco et al. 2016), a question that is not always easy to answer (Wayne and Shaffer 2016).

Our improved understanding of the importance of hybridization in primate evolution has been possible as a result of the rapid development of genetic and genomic approaches (e.g., Papio baboons: Rogers et al. 2018). These methods permit analyzing the phylogenetic relationships among closely related taxa using a multilocus approach, and the signatures of admixture (the mixing of genomes of different taxa through hybridization) and introgression (the passing of alleles from one parental population to another through hybridization and backcrossing). Several primate studies have reported introgression of neutral (e.g., baboons: Wall et al. 2016; howler monkeys: Baiz et al. 2018) and advantageous alleles (e.g., Neanderthal/Denisovan into modern humans: Dannemann et al. 2016; Dolgova and Lao 2018; Enard and Petrov 2018; Gittelman et al. 2016; Racimo et al. 2015, 2017) as a consequence of current or ancient hybridization. In some instances, alleles of only a few genes or genomic regions have moved from one species to another, but in other cases introgression has led to the formation of lineages of hybrid origin. In the case of the kipunji (Rungwecebus kipunji), mitochondrial capture (the replacement of the mitochondrial genome of one species with that of another as a consequence of rare hybridization events) has been considered to explain the presence of baboon-like mitochondrial genomes in one of the two kipunji populations and the presence of kipunji-like mitochondrial genomes in a small yellow baboon (Papio cynocephalus) population adjacent to the range of the other kipunji population (Roberts et al. 2010; Zinner et al. 2009a, 2018). In baboons, phylogenetic analyses also indicate nuclear swamping events (Zinner et al. 2009b), in which the nuclear genome of one species is mostly replaced with that of another species through recurring sex-biased hybridization and introgression. In these cases, extensive nuclear introgression results from the periodic dispersal of either males or females of one (the migrating) taxon into the range of a second (the resident) taxon, with interbreeding that produces hybrid offspring that preferentially backcrosses with members of the immigrating taxon. This swamping of the nuclear genome leads to a phenotypic shift of the resident taxon toward the phenotype of the migrating taxon, and the retention of either the mitochondrial genome of the resident taxon in the case of male-mediated introgression, or the Y-chromosome in the case of female-mediated introgression.

Studies of primate hybridization have also identified alleles that do not cross species boundaries, representing loci associated with reproductive isolation or linked to genomic regions that hold these loci (Sankararaman et al. 2014), and addressed the processes that maintain or strengthen reproductive barriers among hybridizing species (Baiz et al. 2018). Other studies have analyzed the effects of hybridization on morphology (e.g., baboons: Ackermann et al. 2006; Ackerman and Bishop 2010; howler monkeys: Kelaita and Cortés-Ortiz 2013; macaques: Ito et al. 2015), physiology (baboons: Charpentier et al. 2008), and behavior (howler monkeys: Ho et al. 2014; baboons: Bergman et al. 2008; Charpentier et al. 2012). While many studies of primate hybridization focus on identifying phylogenetic incongruences (e.g., among nuclear 
and mitochondrial phylogenies) to test for ancient or ongoing gene flow among lineages, studies of current hybridization and hybrid zones provide deeper insights into evolutionary and ecological processes, including which ecological factors and adaptations allow hybrids to be more successful than parental species, make hybrid speciation likely, or govern the direction of introgression.

In this Special Issue, we compiled eight papers that span behavioral, ecological, and evolutionary topics illustrating the range of factors, processes, and mechanisms that affect primate hybridization. The first contribution, by Roos et al. (2019), illustrates the use of multilocus phylogenetic approaches to identify hybridization during the evolutionary history of a taxon and how admixture may affect the taxonomic designations of species and the interpretation of the evolutionary relationships among taxa. They broaden the taxonomic sampling of Trachypithecus taxa to assess previously observed discordance between phylogenetic inferences based on mitochondrial DNA (mtDNA) and phenotype and nuclear DNA in T. crepusculus, and to test the hypothesis that this taxon has a hybrid origin. Their results strongly support a hybrid origin of T. crepusculus, which has a mitochondrial genome distantly related to the $T$. francoisi group but a nuclear genome more closely related to $T$. barbei, which may have occurred as a consequence of nuclear swamping through male-mediated gene flow from T. barbei to the lineage leading to T. crepusculus.

The second contribution in this Special Issue, by Detwiler (2019), also has an evolutionary perspective and presents a population genetic analysis based on mtDNA sequences of Cercopithecus mitis doggetti and C. ascanius schmidti. The results suggest that historical and contemporary gene flow occurred in the form of unidirectional introgression between the sympatric guenon species, most likely as a consequence of the range expansion of $C$. mitis doggetti into the range of $C$. ascanius schmidti, where male $C$. mitis doggetti reproduced successfully with female $C$. ascanius schmidti, leading to nuclear swamping, such that there are phenotypic $C$. mitis doggetti individuals carrying mitochondrial haplotypes of C. ascanius schmidti.

In the third contribution, Wango et al. (2019) investigated the role of ecological variables as potential barriers to gene flow between Papio anubis and P. cynocephalus in Kenya. Their analysis of various climate and topological variables suggests that the range of $P$. cynocephalus in Kenya is climatically distinct from the range of $P$. anubis, and that hybrids occupy intermediate regions. However, they could not identify a clear pattern of climate or land cover differentiation near the hybrid zone itself. They found that, where both species come into contact, the composition of the resulting population is only weakly predicted by ecological factors (land cover, altitude, climate), and concluded that more fine-grained analyses of these factors may be necessary to detect clear signals of ecological barriers to gene flow.

The fourth contribution, by Soto-Calderón et al. (2018), ${ }^{1}$ presents evidence of hybridization in captivity between Saguinus leucopus and S. oedipus, and between $S$. leucopus and $S$. geoffroyi, using genetic and phenotypic data. This study provides evidence for postzygotic reproductive isolation between closely related tamarin species, as well as the effect of hybridization on the phenotype of these species. Although

\footnotetext{
${ }^{1}$ This article was intended for inclusion in this Special Issue. It was, however, published in a separate issue, Volume 39, Issue 6 and is available at https://doi.org/10.1007/s10764-018-0044-8.
} 
crosses between these three species can produce at least some viable hybrid offspring, the cross between $S$. leucopus and $S$. oedipus suggests the occurrence of gonadal dysgenesis, which will render the observed hybrid individual infertile. Saguinus leucopus and $S$. oedipus have parapatric ranges in northern Colombia, but it is still unknown whether these species hybridize in the wild.

The fifth contribution, by Malukiewicz (2019), reviews instances of hybridization among several species of marmosets (genus Callithrix), and emphasizes the different genomic consequences of natural and anthropogenic hybridization. She synthesizes experimental hybridization studies among five species of marmosets (C. jacchus, C. flaviceps, C. aurita, C. kuhlii, and C. geoffroyi), in which a few hybrid individuals survived, but most showed developmental abnormalities, reduced survival, or were inviable. Malukiewicz then presents a detailed account of all reported instances of natural hybridization within the genus Callithrix, which include five species pairs, and of the admixture between exotic C. jacchus and C. penicillata with native species in southeastern Brazil. This anthropogenic driven hybridization commonly forms "hybrid swarms," where reproductive isolation is not present between parental species and hybrids interbreed among them and backcross with the parental species creating a population with highly admixed individuals. She identifies the need for quantitative analyses of introgression in marmosets, as well as studies to understand the implications of anthropogenic hybridization for the survival and evolution of Callithrix species.

The sixth contribution, by Mourthe et al. (2019), presents the first genetic evidence of current hybridization between South American howler monkeys, Alouatta caraya and A. guariba clamitans, and uses this system to address the hypothesis originally presented by Crockett (1987) that mate recognition drove the evolution of sexual dichromatism in these species through sexual selection. The authors collected samples from regions where hybridization had been hypothesized based on pelage coloration patterns of individuals living in close proximity to populations of both parental species, and analyzed markers of uni- and biparentally inherited loci to determine individual admixture. They found evidence for bidirectional hybridization and putative viability of F1 hybrids. They consider that the observed hybridization between these species with striking interspecific differences in male coat color does not support the argument that sexual selection via mate recognition could have driven the evolution of sexual dichromatism in these species.

The seventh contribution, by Cortés-Ortiz et al. (2019), offers insight into the genomic regions associated with reproductive isolation between two Mexican howler monkey species, Alouatta palliata and A. pigra. They present an extended genetic analysis of patterns of hybridization and introgression in the Mexican howler monkey hybrid zone, using genetic markers from different genomic regions, including the sex chromosomes, autosomes, and mitochondria. They found that mitochondrial haplotypes and alleles of autosomal markers introgress in both directions, but those located on the X-chromosome have limited or no introgression, and the Y-chromosome marker analyzed does not introgress into the heterospecific genomic background. These differential patterns of introgression are consistent with the idea that not all genomic regions introgress uniformly and that, in general, sex chromosomes have a larger contribution to reproductive isolation than autosomal regions. 
In the last article of this Special Issue, Kitchen et al. (2019) analyze the effect of genetic ancestry and experience on acoustic and temporal features of male howler monkey loud calls. The study analyzed vocalizations of unadmixed individuals of the parental species in allopatry and in sympatry, as well as those of male hybrids. The results suggest a strong effect of ancestry for the acoustic features of the calls, whereas the temporal features tended to converge in sympatry, implying some level of plasticity. This study demonstrates that hybrid zones are really "natural laboratories for evolutionary studies," as Godfrey M. Hewitt suggested in 1988 (p. 158).

The compilation of works in this Special Issue represents varied approaches to the study of hybridization and hybrid zones in primates. They help us to understand both the outcomes of hybridization and the factors that affect these outcomes, and the use of well-studied hybrid zones as "natural evolutionary experiments" to address questions about primate behavior that could not be tested in regular experiments because of ethical and logistic limitations. This Special Issue also represents an effort to assemble studies of primate hybridization that are usually scattered in the literature of very different fields because of the multidisciplinary nature of hybridization and hybrid zone research. The studies presented here provide a small window onto the great range of possibilities for studying hybridization (e.g., identification of ancient geneflow between current species, assessment of current processes and mechanism shaping a hybrid zone, or effects of current gene flow on the phenotype) and its implications for primate evolution. It is our hope that this Special Issue will stimulate interest in further studies of hybridization and hybrid zones across primate taxa.

Acknowledgments We thank Joanna Setchell for her support to realize this Special Issue and all the people who reviewed manuscripts submitted to this Special Issue. We are also grateful to all the participants of our symposium at the joint meeting of the International Primatological Society and the American Society of Primatologists, held on August 21-27, 2016 in Chicago for their excellent presentations, constructive comments, and lively discussions. The National Science Foundation (grant BCS-1517701) supported L. Cortés-Ortiz during preparation of this Special Issue.

\section{References}

Abbott, R., Albach, D., Ansell, S., Arntzen, J. W., Baird, S. J. E., et al (2013). Hybridization and speciation. Journal of Evolutionary Biology, 26(2), 229-246.

Ackermann, R. R., \& Bishop, J. M. (2010). Morphological and molcular evidence reveals recent hybridization between gorilla taxa. Evolution, 64(1):271-290.

Ackermann, R. R., Rogers, J., \& Cheverud, J. M. (2006). Identifying the morphological signatures of hybridization in primate and human evolution. Journal of Human Evolution, 51(6), 632-645.

Allendorf, F. W., Leary, R. F., Spruell, P., \& Wenburg, J. K. (2001). The problems with hybrids: Setting conservation guidelines. Trends in Ecology \& Evolution, 16(11), 613-622.

Arnold, M. L., \& Meyer, A. (2006). Natural hybridization in primates: One evolutionary mechanism. Zoology, 109, 261-276.

Baiz M. D., Tucker, P. K., Cortés-Ortiz L. (2018). Multiple forms of selection shape reproductive isolation in a primate hybrid zone. Molecular Ecology, 1-14. https://doi.org/10.1111/mec.14966.

Barton, N. H., \& Hewitt, G. M. (1985). Analysis of hybrid zones. Annual Review of Ecology and Systematics, $16,113-148$.

Bergey, C. M. (2015). Population genomics of a baboon hybrid zone. New York University: PhD thesis.

Bergman, T. J., Phillips-Conroy, J. E., \& Jolly, C. J. (2008). Behavioral variation and reproductive success of male baboons (Papio anubis $\times$ Papio hamadryas) in a hybrid social group. American Journal of Primatology, 70, 136-147. 
Charpentier, M. J. E., Tung, J., Altmann, J., \& Alberts, S. C. (2008). Age at maturity in wild baboons: Genetic, demographic, and environmental influences. Molecular Ecology, 17, 2026-2040.

Charpentier, M. J. E., Fontaine, M. C., Cherel, E., Renoult, J. P., Jenkins, T., et al (2012). Genetic structure in a dynamic baboon hybrid zone corroborates behavioral observations in a hybrid population. Molecular Ecology, 21, 715-731.

Cortés-Ortiz, L., Nidiffer, M. D., Hermida-Lagunes, J., García-Orduña, F., Rangel-Negrín, A., et al (2019). Reduced introgression of sex chromosome markers in the Mexican howler monkey (Alouatta palliata $\times$ A. pigra) hybrid zone. International Journal of Primatology. https://doi.org/10.1007/s10764-018-0056-4.

Crockett, C. M. (1987). Diet, dimorphism and demography: Perspectives from howlers to hominids. In W. G. Kinzey (Ed.), The evolution of human behavior: Primate models (pp. 115-135). Albany: SUNY Press.

Dannemann, M., Andre's, A. M., \& Kelso, J. (2016). Introgression of Neanderthal and Denisovan-like haplotypes contributes to adaptive variation in human toll-like receptors. American Journal of Human Genetics, 98, 22-33.

Detwiler, K. M. (2019). Mitochondrial DNA analyses of Cercopithecus monkeys reveal a localized hybrid origin for C. mitis doggetti in Gombe National Park, Tanzania. International Journal of Primatology. https://oi.org/10.1007/s10764-018-0029-7.

Detwiler, K. M., Burrell, A. S., \& Jolly, C. J. (2005). Conservation implications of hybridization in African cercopithecine monkeys. International Journal of Primatology, 26(3), 661-684.

Dolgova, O., \& Lao, O. (2018). Evolutionary and medical consequences of archaic introgression into modern human genomes. Genes, 9(7), 358.

Enard, D., \& Petrov, D. A. (2018). Evidence that RNA viruses drove adaptive introgression between Neanderthals and modern humans. Cell, 175(2), 360-371.

Gittelman, R. M., Schraiber, J. G., Vernot, B., Mikacenic, C., Wurfel, M. M., \& Akey, J. M. (2016). Archaic hominin admixture facilitated adaptation to Out-of-Africa environments. Current Biology, 26(24), 33753382.

Hewitt, G. M. (1988). Hybrid zones: Natural laboratories for evolutionary studies. Trends in Ecology \& Evolution, 3(7), 158-167.

Ho, L., Cortés-Ortiz, L., Dias, P. A. D., Canales-Espinosa, D., Kitchen, D. M., \& Bergman, T. J. (2014). Effect of ancestry on behavioral variation in two species of howler monkeys (Alouatta pigra and A. palliata) and their hybrids. American Journal of Primatology, 76(9):855-867.

Ito, T., Kawamoto, Y., Hamada, Y., \& Nishimura, T. D. (2015). Maxillary sinus variation in hybrid macaques: implications for the genetic basis of craniofacial pneumatization. Biological Journal of the Linnean Society, 115(2):333-347.

Jolly, C. J. (1993). Species, subspecies, and baboon systematics. In W. H. Kimbel \& L. B. Martin (Eds.), Species, species concepts, and primate evolution (pp. 67-101). New York: Plenum Press.

Kearns, A. M., Restani, M., Szabo, I., Schrøder-Nielsen, A., Kim, J. A., et al (2018). Genomic evidence of speciation reversal in ravens. Nature Communications, 9(1), 906.

Kelaita, M. A., \& Cortés-Ortiz, L. (2013). Morphological variation of genetically confirmed Alouatta pigra $\times$ A. palliata hybrids from a natural hybrid zone in Tabasco, Mexico. American Journal of Physical Anthropology, 150(2), 223-234.

Kitchen, D. M., Bergman, T. J., Dias, P. A. D., Ho, L., Canales-Espinosa, D., \& Cortés-Ortiz, L. (2019). Temporal but not acoustic plasticity in hybrid howler monkey (Alouatta palliata $\times$ A. pigra) loud calls. International Journal of Primatology. https://doi.org/10.1007/s10764-017-0004-8.

Malukiewicz, J. (2019). A review of experimental, natural, and anthropogenic hybridization in Callithrix marmosets. International Journal of Primatology. https://doi.org/10.1007/s10764-018-0068-0.

Malukiewicz, J., Boere, V., Fuzessy, L. F., Grativol, A. D., de Oliveira e Silva, I., et al (2015). Natural and anthropogenic hybridization in two species of Eastern Brazilian marmosets (Callithrix jacchus and $C$. penicillata). PLoS ONE 10(6): $\mathrm{e} 0127268$.

Mourthe, I., Trindade, R. A., Aguiar, M., Trigo, T. C., Bicca-Marques, J. C., \& Bonatto, S. L. (2019). Hybridization between Neotropical primates with contrasting sexual dichromatism. International Journal of Primatology. https://doi.org/10.1007/s10764-017-0011-9.

Racimo, F., Sankararaman, S., Nielsen, R., \& Huerta-Sánchez, E. (2015). Evidence for archaic adaptive introgression in humans. Nature Reviews Genetics, 16, 359.

Racimo, F., Marnetto, D., \& Huerta-Sánchez, E. (2017). Signatures of archaic adaptive introgression in present-day human populations. Molecular Biology and Evolution, 34(2), 296-317.

Ridley, M. (2004). Evolution. Oxford: Blackwell.

Roberts, T. E., Davenport, T. R. B., Hildebrandt, K. B. P., Jones, T., Stanley, W. T., et al (2010). The biogeography of introgression in the critically endangered African monkey Rungwecebus kipunji. Biology Letters, 6(2), 233-237. 
Rogers, J., Raveendran, M., Harris, R. A., Mailund, T., Leppälä, K., et al. (2018). The comparative genomics and complex population history of Papio baboons. Science Advances. (in press).

Roos, C., Liedigk, R., Thinh, V., Nadler, T., \& Zinner, D. (2019). The hybrid origin of the Indochinese gray langur Trachypithecus crepusculus. International Journal of Primatology. https://doi.org/10.1007 /s10764-017-0008-4.

Sankararaman, S., Mallick, S., Dannemann, M., Prüfer, K., Kelso, J., et al (2014). The landscape of Neandertal ancestry in present-day humans. Nature, 507(7492), 354-357.

Seehausen, O. (2006). Conservation: Losing biodiversity by reverse speciation. Current Biology, 16(9), PR334-PR337.

Soto-Calderón, I. D., Acevedo-Garcés, Y. A., Restrepo-Agudelo, T., Llinás-Guerrero, J. C., Rivillas-Puello, Y., \& López, J. B. (2018). Phenotypic, genetic, and cytogenetic evidence of hybridization between species of Trans-Andean tamarins (genus Saguinus). International Journal of Primatology, 39(6), 1022-1038.

Todesco, M., Pascual, M. A., Owens, G. L., Ostevik, K. L., Moyers, B. T., et al (2016). Hybridization and extinction. Evolutionary Applications, 9(7), 892-908.

Tung, J., \& Barreiro, L. B. (2017). The contribution of admixture to primate evolution. Current Opinion in Genetics \& Development, 47, 61-68.

Tung, J., Charpentier, M. J., Mukherjee, S., Altmann, J., \& Alberts, S. C. (2012). Genetic effects on mating success and partner choice in a social mammal. The American Naturalist, 180, 113-129.

Wall, J. D., Schlebusch, S. A., Alberts, S. C., Cox, L. A., Snyder-Mackler, N., et al (2016). Genome-wide ancestry and divergence patterns from low-coverage sequencing data reveal a complex history of admixture in wild baboons. Molecular Ecology, 25(14):3469-3483.

Wango, T. L., Musiega, D., Mundia, C. N., Altmann, J., Alberts, S. C., \& Tung, J. (2019). Climate and land cover analysis suggest no strong ecological barriers to gene flow in a natural baboon hybrid zone. International Journal of Primatology. https://doi.org/10.1007/s10764-017-9989-2.

Wayne, R. K., \& Shaffer, H. B. (2016). Hybridization and endangered species protection in the molecular era. Molecular Ecology, 25, 2680-2689.

Zinner, D., Arnold, M. L., \& Roos, C. (2009a). Is the new primate genus Rungwecebus a baboon? PLoS ONE, 4(3), e4859.

Zinner, D., Groeneveld, L. F., Keller, C., \& Roos, C. (2009b). Mitochondrial phylogeography of baboons (Papio spp.): Indication for introgressive hybridization? BMC Evolutionary Biology, 9(1), 83.

Zinner, D., Arnold, M. L., \& Roos, C. (2011). The strange blood: Natural hybridization in primates. Evolutionary Anthropology, 20(3), 96-103.

Zinner, D., Chuma, I. S., Knauf, S., \& Roos, C. (2018). Inverted intergeneric introgression between critically endangered kipunjis and yellow baboons in two disjunct populations. Biology Letters, 14(1). https://doi. org/10.1098/rsbl.2017.0729. 